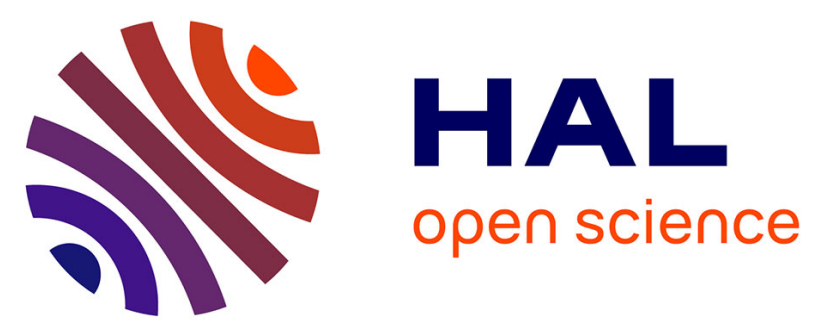

\title{
Carbapenemase-producing Enterobacteriaceae digestive carriage at hospital readmission and the role of antibiotic exposure
}

\author{
S. Evain, C. Bourigault, M.-E. Juvin, S. Corvec, D. Lepelletier
}

\section{To cite this version:}

S. Evain, C. Bourigault, M.-E. Juvin, S. Corvec, D. Lepelletier. Carbapenemase-producing Enterobacteriaceae digestive carriage at hospital readmission and the role of antibiotic exposure. Journal of Hospital Infection, 2019, 102, pp.25 - 30. 10.1016/j.jhin.2019.02.002 hal-03485081

\section{HAL Id: hal-03485081 \\ https://hal.science/hal-03485081}

Submitted on 20 Dec 2021

HAL is a multi-disciplinary open access archive for the deposit and dissemination of scientific research documents, whether they are published or not. The documents may come from teaching and research institutions in France or abroad, or from public or private research centers.
L'archive ouverte pluridisciplinaire HAL, est destinée au dépôt et à la diffusion de documents scientifiques de niveau recherche, publiés ou non, émanant des établissements d'enseignement et de recherche français ou étrangers, des laboratoires publics ou privés.

\section{(ㄷ)(1) $\$$}

Distributed under a Creative Commons Attribution - NonCommerciall 4.0 International 


\section{Title page}

\section{Title}

Carbapenemase-producing Enterobacteriaceae (CPE) digestive carriage: How many carriers are already positive at hospital readmission and what is the role of antibiotic exposure for the remaining positive patients during hospital stay?

\section{Authors}

Sullivan EVAIN ${ }^{1}$, Céline BOURIGAULT ${ }^{1,2}$, Marie-Emmanuelle JUVIN ${ }^{2}$, CORVEC Stéphane $^{2}$, LEPELLETIER Didier ${ }^{1,2^{*}}$

\section{Affiliation}

${ }^{1}$ Microbiotas, Host, Antibiotics, bacterial Resistance (MiHAR Lab) (www.mihar.univnantes.fr), University of Nantes, Nantes, FR-44000

${ }^{2}$ Bacteriology and Infection Control Department, Nantes University Hospital, Nantes, FR44000

\section{Corresponding author}

*Prof. Didier Lepelletier (mail: didier.lepelletier@ chu-nantes.fr), MiHAR Lab

(www.mihar.univ-nantes.fr), University of Nantes, Nantes, 5 rue du Professeur Yves Boquien,

Bât le Tourville, FR-44093 Cedex 1. International phone number (+33) 611915233. 


\section{Summary}

Introduction. The aims of our study were to identify how many prior OXA-48 carbapenemase-producing Enterobacteriaceae (CPE) positive patients identified during an outbreak period were readmitted, to evaluate their CPE positive or negative digestive tract colonization at hospital readmission and during readmission stay and to assess the role of antibiotic exposure on their CPE colonization status during readmission.

Method. All CPE cohort patients from June 2013 to May 2016 (N=189) were registered in a survey database and were systematically identified at readmission by a daily informatics and alert program using specific hospital population number. Each cohort patient was systematically screened for CPE colonization at the day of readmission and then weekly if the length of stay was longer than 6 days.

Results. 114 (60.3\%) patients previously CPE colonized were readmitted to our hospital. Excluding the 12 patients who were not screened because their period of readmission was $<24$ h, 88 patients were negative $(86.3 \%)$ and 14 were positive $(13.7 \%)$ for CPE colonization at first hospital readmission. The 14 CPE-positive patients didn't change their infectious status and remained CPE-positive during the study period. Of the 88 negative patients, 65 remained negative during the study period, and 23 subsequently became CPE-positive after the negative readmission screening. CPE positive colonization was significantly associated with antibiotic exposure during readmission periods $(\mathrm{P}<0.001)$.

Conclusion. Negative screens at hospital readmission did not necessary predict resolution of CPE carriage. Antibiotic exposure appears to influence the risk of remaining CPE-positive. 


\section{Introduction}

Our hospital experienced a large outbreak caused by Klebsiella pneumoniae producing OXA48 carbapenemase between June 2013 and June 2015 [1]. The first case retrospectively identified was a patient repatriated from Morocco after a quad motor accident. This outbreak involved several healthcare wards and generated more than 180 secondary cases during the outbreak period, and was managed using French guidelines [2]. Duration of colonization by carbapenemase-producing Enterobacteriaceae (CPE) can persist several months after hospital discharge especially in patient with altered microbiota [3-5]. These findings closely affect the clinical management and infection control measures at the time of CPE colonized patients readmission. The aims of our study were to identify how many prior CPE positive patients were readmitted, to evaluate their CPE positive or negative colonization at hospital readmission and to assess the role of antibiotic exposure on their positive CPE colonization during readmission.

\section{Methods}

\section{Setting and study design}

The study was a single center survey performed at the Nantes University Hospital, France. Our hospital is a 3,000-bed teaching hospital providing all acute-cares with around 90,000 admissions and 900,000 hospital-days per year, respectively. We implemented a retrospective analysis of a cohort CPE colonized patients detected during a previous outbreak period [1] when readmitted at the Nantes University Hospital from February 2013 to May 2016.

\section{Patients}

All cohort patients were registered in a survey database and were systematically identified at readmission by a daily informatics and alert program using specific hospital population number. Each cohort patient was systematically screened for CPE colonization at the day of readmission and then weekly if the length of stay was longer than 6 days. Number of CPE 
positive or negative patients was calculated according to the time between prior admission and first readmission ( $<3$ months, $<6$ months, $<12$ months, $<18$ months and $>18$ months).

\section{Microbiology}

Stool samples were collected with rectal swab and cultured on selective chromogenic media (chromID CARBA-SMART ID®). MASTDISCS ESBL-E and MASTDISC- carbapenemase (CARB / OXA48) were used to confirm the suspected resistance phenotype.

\section{Collection of data}

Demographic, clinical, microbiological (result of systematic screening for CPE colonization at each readmission, characterization of $\mathrm{CPE}$ ), antibiotic exposure during readmission periods and intra-hospital death were collected from the electronic medical records. Each patient could be readmitted several times in our hospital during the study period (ie. cares for chronic diseases). Each patient was studied to assess the CPE colonization status at readmission and antibiotic exposure. We defined patients without change in their CPE infectious status (negative or positive) and those with $\mathrm{CPE}$ infectious status that have changed during readmission periods (negative to positive). Data according to demographic characteristics and to CPE carriage at first hospital readmission were related to each readmitted patient; data according to the length of stay, antibiotic exposure, and to CPE carriage classification (positive/positive or negative/positive) were related to each hospital readmission.

\section{Statistics}

Data were collected using Excel® software. Data were described using the mean with standard deviation (SD) for continuous variables and proportions (\%) for qualitative variables. Comparisons relied on the Kruskal-Wallis test for continuous variables and on the chi-square test or Fisher's exact test, as appropriate, for qualitative variables. All P values were two sided, and $\mathrm{P}$ values $<0.05$ were considered significant. 
We used the STROB (STrengthening the Reporting of OBservational studies in Epidemiology) criteria for transparent reporting [6]. 


\section{RESULTS}

In total, 189 patients were detected positive for CPE digestive carriage during the outbreak period (Jun 2013 - June 2015) with the clonal strain. The median age was 66.6 years $( \pm 16.6$ SD) and 58\% were male. The microbiological analysis showed monobacterial results in 142 cases: the most common Enterobacteriaceae producing OXA-48 were K. pneumoniae $(61 / 142,43 \%)$ and E. coli $(58 / 142,40.8 \%)$. Among other samples $(\mathrm{N}=47)$, two different OXA-48 were detected, mainly E. coli and K. pneumonia (Figure 1). Molecular analysis showed a clonal spread of both strains and plasmids. The majority of these identifications were made by systematic epidemiological rectal screening for EPC OXA-48 colonization with culture exam $(166 / 189,87.8 \%)$. In the other cases, they were performed from clinical specimens such as cytological and bacteriological urinary exam $(11 / 189,5.8 \%)$, blood culture $(5 / 189,2.6 \%)$, the combination of $2(1 / 189,0.5 \%)$, rapid real-time PCR screening (4/189, $2.1 \%)$, cytological and bacteriological sputum test $(1 / 189,0.5 \%)$ or surgical wound $(1 / 189$, $0.5 \%)$

During this study period, 47/189 (24.9\%) CPE positive carriers died. Among them, 19 (40.4\%) deaths occurred during the first hospitalization (detection of the first CPE colonization). Around 3,000 contact patients were detected during the outbreak period and were included in a specific alert database in order to screen them if readmitted in our hospital. During the study period, 500 contact patients were readmitted (17\%) and 5 of them were found to be positive for OXA-48 CPE colonization, suggesting cross transmission during their hospitalization $(1 \%)$.

During this study period, 114 (60.3\%) patients previously CPE colonized were readmitted to our hospital. Of these $114 \mathrm{CPE}$ colonized patients, $67.5 \%$ of them were readmitted more than once. In total, we analyzed 359 readmissions with a median number of 2 (range 1-13). The median time between 2 readmissions was 36 days (range 0-712). Patients were mainly 
readmitted in three healthcare wards: infectious diseases $(33.9 \%)$, immunology-nephrology ward (19.6\%) and internal medicine departments (17.5\%), respectively. Among the 359 readmissions, the length of stay varied from $<5$ days (21.4\%), between 5 and 10 days (39.3\%), between 11 and $20(25.9 \%)$ and $>21(13.6 \%)$.

Excluding the 12 patients who were not screened instead the protocol (readmission $<24 \mathrm{~h}$ ), 88/102 (86.3\%) patients were negative and $14(13.7 \%)$ were positive for CPE colonization at first hospital readmission, respectively. Those $14 \mathrm{CPE}$ positive patients didn't change their infectious status and remained CPE positive during the study period. Of the 88 negative patients, 65 remained negative during the study period and 23 were detected CPE positive after the negative readmission screening (Figures 2 and 3). A total of 472 swabs were made for all the 359 readmissions during the study period and $65.5 \%$ were negative for CPE colonization (309/472).

Forty four percent of patients were exposed to antibiotics during readmission (50/114). The rate of readmission with antibiotic exposure was $24.8 \%$ (89/359). The median duration of antibiotic exposure was 6.5 days (range 1-55). The median number of antibiotics per patient was 2 (1-9): 43 antibiotics of 25 different classes were prescribed including carbapenems and trimethoprim-sulfamethoxazole $(31 / 89,34.8 \%$, respectively), amoxicillin-clavulanic acid $(29 / 89,32.6 \%)$ and cephalosporins $(26 / 89,29.0 \%)$. Sixteen cohort patients $(14.0 \%)$ were never exposed to antibiotics during readmissions and 13 always (11.4\%).

CPE positive colonization was significantly associated with antibiotic exposure during readmission periods ( $\mathrm{P}<0.001)$ (Figure 2). 


\section{DISCUSSION}

In this study, we demonstrated the low level of CPE positive patient at hospital readmission and the role of antibiotic exposure. We found that only $14 \%$ of patients with past history of CPE colonization remained positive at the time of the first hospital readmission. But $20 \%$ of patients detected negative at readmission were again positive for CPE gastrointestinal carriage either during the first readmission if $>6$ days or during new readmissions. We studied the relation between CPE positive/negative gastrointestinal colorization and the antibiotic exposure. We found a significant association between antibiotic exposure and the CPE positivity in patients identified as testing CPE negative at the first readmission. This finding highlights the role of antibiotics in the microbiota modification and the ability to detect CPE carriage. Antibiotics with high activity against anaerobes that were mostly used in our studied population potently affect the capacity of the microbiota to prevent colonization by exogenous microorganisms with an increase of relative abundance of resistant enterobacteria [7]. All CPE positive patients are at risk to transmit CPE to patients hospitalized in the same ward by cross-transmission through health cares and environment [8]. In this situation, barrier precautions and systematic gastrointestinal screening is needed among contact patients to evaluate the possible cross-transmission during all the hospitalization of CPE positive patient [2].

Patients were mostly detected CPE positive at hospital readmission within a period of three or 12 months after their initial hospitalization and rarely after 12 months. In contrast, most of the patients $(60 \%)$ were CPE negative at hospital readmission. Without antibiotic exposition, these patients with previous history of positive CPE gastrointestinal carriage can be considered at lower risk for CPE transmission as they mostly remained negative. This nondetection of CPE gastrointestinal carriage at readmission is probably related to the low rectal CPE concentrations of these patients as a weak linear correlation between the degree of 
gastrointestinal carriage of $\mathrm{CPE}$ and environmental contamination has been previously described [8]. Those results allow us to propose to survey those low risk patients without isolation precaution after two negative screenings during the same hospital stay of $>6$ days. We recommend therefore performance of an additional rectal swab 48 or 72 hours after a new antibiotic exposure. We also recommend systematic screening of them again at each readmission time, as microbiota modification can occurred between two hospitalizations (community health cares or antibiotic exposure). But we don't know actually how much time and after how many successive negative screenings we need to survey (one year, all the life for patients with multiple readmissions for chronic diseases) as negative patients were already detected again positive for CPE colonization after 5 negative screenings $[9,10]$. Accurate and reliable determination of definitive negativity of CPE gastrointestinal carriage is important for both patients and healthcare settings, especially when an active strategy of carrier surveillance is performed. Our results show that a single negative rectal swab is probably not sufficient to state of the definitive negative status of previous CPE colonized patients, as 23 negative patients at the time of readmission with more than two tests did have a subsequent positive screening. However, the total number of tests per patient and the duration of follow-up in our study are probably not sufficient to determine the exact number of tests that are required for defining CPE clearance according to the discontinuing carrier follow-up [3]. However, our risk factor analyses can indicate patients that are less likely to remain carriers (patients with several negative screening and without antibiotic exposure).

By the cohort contact patient's follow-up during our study period, we found that very few readmitted contact patients were at risk of carriage as only $1 \%$ of them were detected positive for CPE gastrointestinal carriage (unpublished data). The risk of positive carriage among contacts is probably related to the initial outbreak situation with more or less secondary cases, the antibiotic exposure during hospital stay and the time between the CPE acquisition and the 
readmission. Also, no data are available to let us know when we can stop surveillance and screening and when we can exclude contact patients from database.

Our results are relevant in this field as few data are available but this study has some limitations. This retrospective analysis of CPE patient cohort is a single centre study. The time between the previous hospitalization and the first readmission was heterogeneous, and patients and contacts not studied may have been hospitalized in other hospitals of the region area and results may have been over or underestimated. Readmitted patients to our university hospital may represent a population with higher risk of CPE carriage because of chronic underlying diseases and more frequent antibiotic exposures. And some previous CPE colonized patients may have not been detected at hospital readmission for microbiological features [11].

Since the implementation of this survey, no secondary cases were detected in our hospital from readmitted at risk patients (contact patients or previous positive colonized patients) (unpublished data). The key factor for this successful control was the daily detection programme by the hospital informatics system, the infection control team automatic alert and the awareness of each healthcare professional of our hospital in implementing isolation and in screening suspected patients immediately at the readmission time. At the time of global menace [12], this surveillance strategy needs daily risk assessment to implement the best preventive precaution to control the CPE spread $[13,14]$.

In conclusion, CPE positive patients are frequently readmitted. Negative screenings at hospital readmission did not necessary predict CPE resolution carriage. ATB exposure appears to influence the risk to remain positive for CPE gastrointestinal colonization. Negative carriers at hospital readmission with several negative tests could be defined as at low risk of transmission, especially when they are not exposed to antibiotics. Prevention policies could take into account this remaining negative CPE colonization status for the 
indication to screen systematically all contact patients. Further studies are needed to better understand and assess the duration and resolution of CPE carriage. 


\section{Conflicts of interest}

The authors declare no competing interests regarding this manuscript.

\section{Funding}

No specific funding has been received.

\section{Contribution}

CB and DL designed the study. SE, CB and SC screened the literature for relevancy which was reviewed by DL. MEJ, did the data extraction and the surveillance of digestive screening at the lab. SE, CB and DL did the quality appraisal. SE and DL drafted the manuscript. CB, $\mathrm{SC}$, and DL revised the manuscript. All authors participated in data interpretation and revised the manuscript for intellectual content. 


\section{REFERENCES}

[1] Semin-Pelletier B, Cazet L, Bourigault C, Juvin ME, Boutoille D, Raffi F, Hourmant M, Blancho G, Agard C, Connault J, Corvec S, Caillon J, Lepelletier D. Challenges of controlling a large outbreak of OXA-48 carbapenemase-producing Klebsiella pneumoniae in a French university hospital. J Hosp Infect 2015;89:248-53.

[2] Lepelletier D, Berthelot P, Lucet JC, Fournier S, Jarlier V, Grandbastien B; National Working Group. French recommendations for the prevention of 'emerging extensively drug-resistant bacteria' (eXDR) cross-transmission. J Hosp Infect 2015;90:186-95.

[3] Feldman N, Adler A, Molshatzki N, Navon-Venezia S, Khabra E, Cohen D, Carmeli Y. Gastrointestinal colonization by KPC-producing Klebsiella pneumoniae following hospital discharge: duration of carriage and risk factors for persistent carriage. Clin Microbiol Infect 2013;19: E190-6.

[4] Rossini A, Di Santo SG, Libori MF, Tiracchia V, Balice MP, Salvia A. Risk factors for carbapenemase-producing Enterobacteriaceae colonization of asymptomatic carriers on admission to an Italian rehabilitation hospital. J Hosp Infect 2016;92:78-81.

[5] Haverkate M, Derde LP, Brun-Buisson C, Bonten M, Bootsma MC. Duration of colonization with antimicrobial-resistant bacteria after ICU discharge. Intensive Care Med 2014; 40:564-571.

[6] von Elm E, Altman DG, Egger M, Pocock SJ, Gøtzsche PC, Vandenbroucke JP; STROBE Initiative. The Strengthening the Reporting of Observational Studies in Epidemiology (STROBE) Statement: guidelines for reporting observational studies. Int J Surg 2014;12:1495-9. 
[7] Ruppé E, Andremont A. Causes, consequences, and perspectives in the variations of intestinal density of colonization of multidrug-resistant enterobacteria. Front Microbiol $2013 ; 28 ; 4: 129$.

[8] Lerner A, Adler A, Abu-Hanna J, Cohen Percia S, Kazma Matalon M, Carmeli Y. Spread of KPC-producing carbapenem-resistant Enterobacteriaceae: the importance of super-spreaders and rectal KPC concentration. Clin Microbiol Infect 2015;21:470.e1-7.

[9] Humphreys H, Prior AR. CPE colonization - Once A carrier always a carrier? Response to Lewis and Bart. Infect Control Hosp Epidemiol 2015;36:1482-3.

[10] Lewis JD, Enfield KB, Mathers AJ, Giannetta ET, Sifri CD. The limits of serial surveillance cultures in predicting clearance of colonization with carbapenemaseproducing Enterobacteriaceae. Infect Control Hosp Epidemiol 2015;36:835-7.

[11] Simner PJ, Martin I, Opene B, Tamma PD, Carroll KC, Milstone AM. Evaluation of multiple methods for detection of gastrointestinal colonization of carbapenem-Resistant organisms from rectal swabs. J Clin Microbiol 2016;54:1664-1667.

[12] Logan LK, Weinstein RA. The Epidemiology of Carbapenem-Resistant Enterobacteriaceae: The Impact and Evolution of a Global Menace. J Infect Dis 2017;215(suppl_1):S28-S36.

[13] French CE, Coope C, Conway L, Higgins JP, McCulloch J, Okoli G, Patel BC, Oliver I. Control of carbapenemase-producing Enterobacteriaceae outbreaks in acute settings: an evidence review. J Hosp Infect 2017;95(1):3-45.

[14] Poole K, George R, Decraene V, Shankar K, Cawthorne J, Savage N, Welfare W, Dodgson A. Active case finding for carbapenemase-producing Enterobacteriaceae in a teaching hospital: prevalence and risk factors for colonization. J Hosp Infect 2016;94:125-9. 


\section{List of figures}

Figure 1. Distribution and number of strains per specimens of the different OXA-48 producing Enterobacteriaceae isolated from the 189 positive patients $(\mathrm{N}=189)$

Figure 2. Flow chart related to the CPE infectious status at hospital readmission and during readmission stay among patients with past history of CPE colonization

Figure 3. Incidence of CPE negative $(\mathrm{N}=65)$ or positive $(\mathrm{N}=14)$ patients at hospital readmission among patients with past history of CPE colonization according to the interval time between the first hospitalization (CPE acquisition) and readmission. 


\section{Figure 1.}

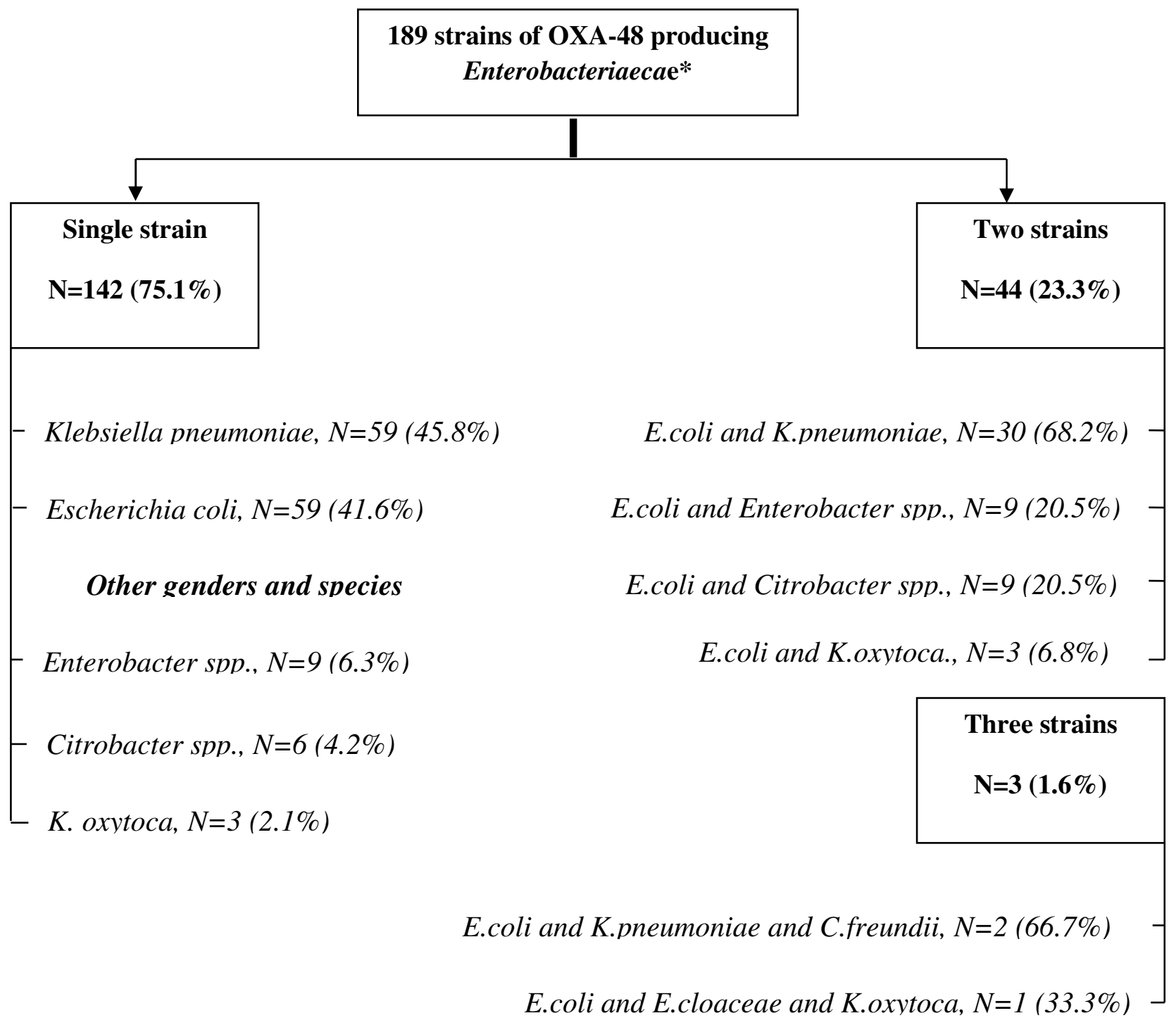


Figure 2.
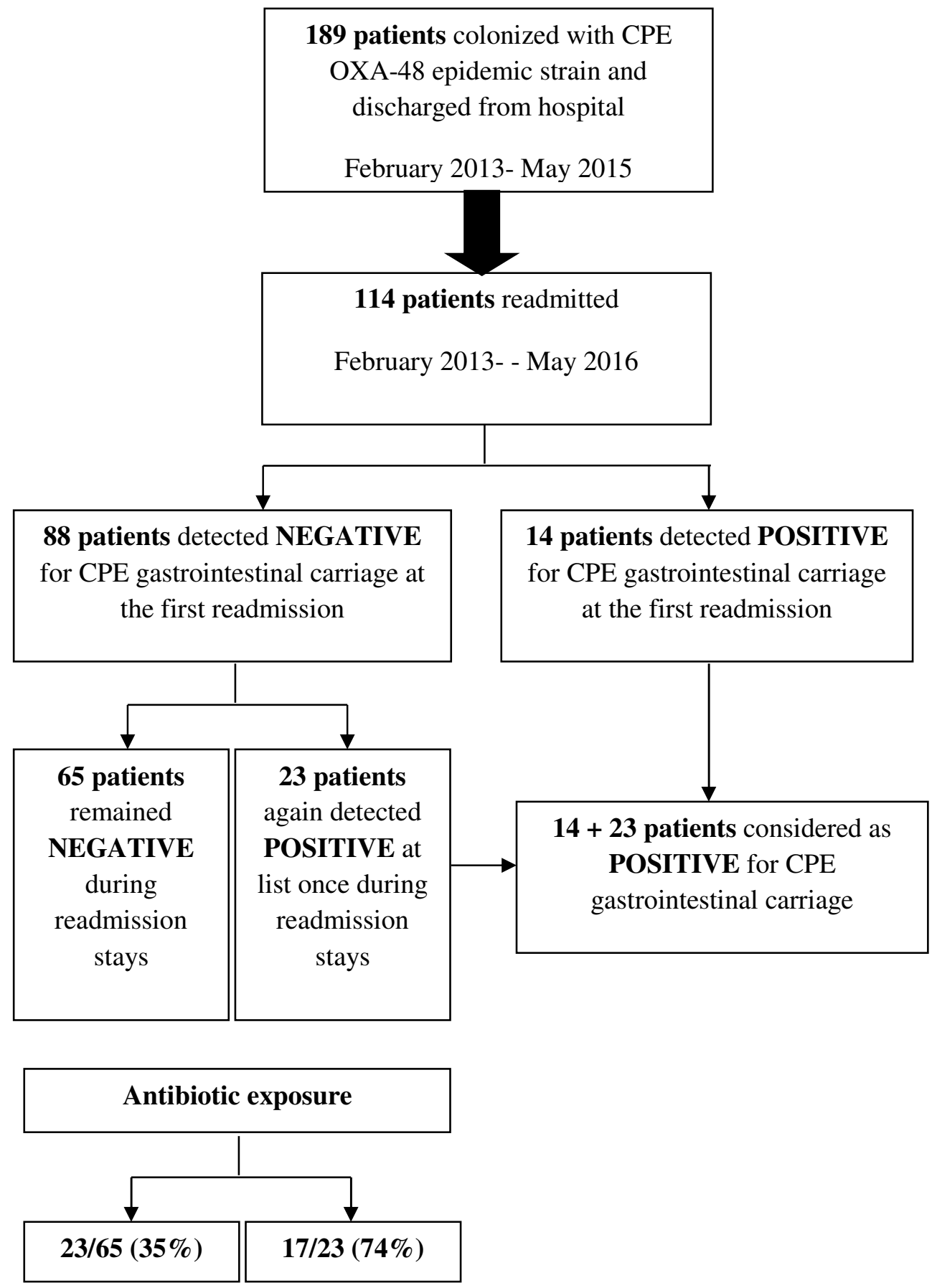
Figure 3.

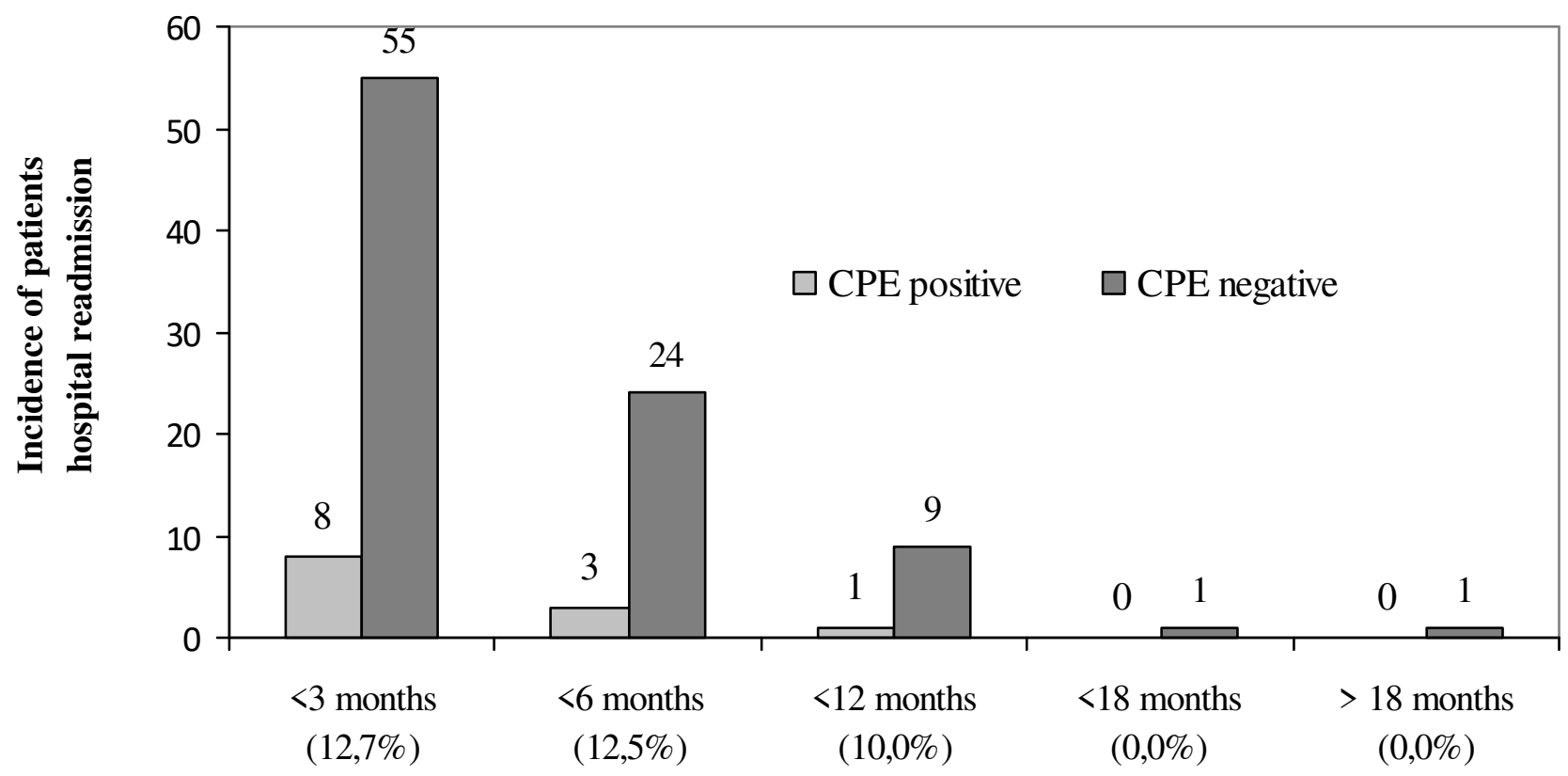

(\%, proportion of CPE positive patients) 